\title{
A HEURISTIC FOR TWO MACHINE OPEN SHOP SPECIALLY STRUCTURED SCHEDULING PROBLEM TO MINIMIZE THE RENTAL COST, INCLUDING JOB BLOCK CRITERIA
}

\author{
Deepak Gupta ${ }^{1}$, Shashi Bala ${ }^{2}$, Payal Singla ${ }^{3 \S}$ \\ $1,2,3$ Department of Mathematics \\ Maharishi Markandeshwar University \\ Mullana, Ambala, 133001, Haryana, INDIA
}

\begin{abstract}
The present paper considers a more practical problem of scheduling $\mathrm{n}$ jobs in a two machine specially structured open shop to minimize the rental cost. Further the processing time of jobs are associated with their respective probabilities including job block criteria. In most of literature the processing times are always considered to be random, but there are significant situations in which processing times are not merely random but bear a well defined structural relationship to one another. The objective of this paper is to minimize the rental cost of machines under a specified rental policy. A numerical illustration is given to support the algorithm.
\end{abstract}

AMS Subject Classification: 90B35, 90B30

Key Words: open shop scheduling, rental policy, processing time, utilization time, makespan, idle time

\section{Introduction}

In a scheduling problems for shop processing system, each job has to be processed on a specific machine. A job passing through the machines following a certain order is known as the processing route. If the processing routes are not given in advance, but have to be chosen, the processing system is called the open shop. If the processing routes are fixed beforehand and are same for all

Received: May 16, 2012

(C) 2013 Academic Publications, Ltd.

$\S$ Correspondence author 
the jobs, the system is called flow shop. Such an open shop environment arise in many industrial application viz, automobile repairs, quality control centres, semiconductor manufacturing, setellite communication etc. Johnson [1] gave a procedure to obtain the optimal sequence for n-jobs, two three machines flow shop scheduling problem with an objective to minimize the makespan. the work was developed by Gupta, J.N.D. [4],Maggu and Dass [9], Gupta Deepak [8], Anup [18], V.A. Strusevich [14,16], Singh T.P. [12], Yoshida and Hitomi [10] etc. by considering various parameter.

Singh T.P and Gupta Deepak [5] made an attempt to study the optimal two stage open shop scheduling in which processing time is associated with their respective probabilities including job block criteria. Gupta Deepak, Sharma and Shashi [20] introduce the concept of specially structured flow shop scheduling to minimize the rental cost of machines. In which processing times are associated with probabilities. The present paper is an attempt to minimize the rental cost of machines by introducing the concept of job restriction under specially structured open shop scheduling. The idea of job restrictions has a practical significance to create a balance between the cost of providing priority in service to customers and cost of giving service with non priority customers thereby the problem becomes wider and more applicable in a production concern.

\section{Practical Situation}

Many applied and experimental situation occur in our day to day working in factories and industrial concern when we have to restrict the processing of some jobs. The practical situation may be taken in a production industry; manufacturing industry etc. where some jobs has to give priority over others. In the era of globalization or global uncertainties, to meet the challenges of the business, one does not always have enough funds to invest in advanced machines to update the technology. Under such circumstances the machines has to be taken on rent. Rental of machines is an affordable and quick solution for having the equipment and up gradation to new technology.

\section{Notations}

$S_{k} \quad$ : Sequence obtained by applying Johnsons procedure, $\mathrm{k}=1,2,3 \ldots$

$M_{j} \quad$ : Machine $\mathrm{j}=1,2$

$a_{i j} \quad$ : Processing time of $i^{t h}$ job on machine $M_{j}$

$p_{i j} \quad$ : Probability associated to the Processing time $a_{i j}$ 
$A_{i j} \quad$ : Expected Processing time

$t_{i j}\left(S_{k}\right)$ : transportation time of $i^{t h}$ job to transpose from one machine to another machine

$U_{i}\left(S_{k}\right)$ : Utilization time for which machine $M_{j}$ is required

$R\left(S_{k}\right)$ : Total rental cost for the sequence $\left(S_{k}\right)$ of all machine

$C T\left(S_{k}\right)$ : Total completion time of the jobs for sequence $\left(S_{k}\right)$

$C_{i} \quad$ : Rental cost of $i^{\text {th }}$ machine

$\beta \quad$ : Equivalent job block.

\section{Definition}

Completion time of $i^{\text {th }}$ job on machine $M_{j}$ is denoted by $t_{i j}$ and is defined as: $t_{i j}=\max \left(t_{i-1, j}, t_{i, j-1}\right)+a_{i j} \mathrm{x} p_{i j}$ for $\mathrm{j} \geq 2$

$=\max \left(t_{i-1, j}, t_{i, j-1}\right)+A_{i j}$, where $A_{i j}=$ Expected processing time of $i^{\text {th }}$ job on $j^{\text {th }}$ machine.

\section{Rental Policy $(\mathrm{P})$}

The machines will be taken on rent as and when they are required and are returned as and when they are no longer required. i.e. the first machine will be taken on rent in the starting of processing the jobs, second machine will be taken on rent at time when first job is completed on the first machine.

\section{Problem Formulation}

Let $\mathrm{n}$ jobs $1,2, . ., \mathrm{n}$ be processed on machines two machines $M_{1}$ and $M_{2}$ in any order i.e. the jobs will be processed first on $M_{1}$ and then on $M_{2}$ or first on $M_{2}$ and then on $M_{1}$ under the specified rental policy. Let $a_{i j}$ be the processing time of $i^{\text {th }}$ job on $(\mathrm{i}=1,2, . ., \mathrm{n})$ on machine $M_{j}$ and $p_{i j}$ be the probabilities associated with $a_{i j}$. $A_{i j}$ be the expected processing time of $i^{\text {th }}$ job on machine $M_{j}(\mathrm{j}=$ $1,2, \mathrm{n})$ such that either $A_{i 1} \geq A_{i 2}$ or $A_{i 1} \leq A_{i 2}$.

Our aim is to find the optimal or near optimal sequence $S_{k}$ of the jobs which minimize the rental cost of machines. The mathematical model of the given problem in matrix form can be stated as: 


\begin{tabular}{|c|c|c|c|c|}
\hline jobs & \multicolumn{2}{|c|}{ Machine } & $\mathrm{A}$ & \multicolumn{2}{c|}{ Machnie } & $\mathrm{B}$ \\
\hline $\mathrm{i}$ & $a_{i 1}$ & $p_{i 1}$ & $a_{i 2}$ & $p_{i 2}$ \\
\hline 1 & $a_{11}$ & $p_{11}$ & $a_{12}$ & $p_{12}$ \\
\hline 2 & $a_{21}$ & $p_{21}$ & $a_{22}$ & $p_{22}$ \\
\hline 3 & $a_{31}$ & $p_{31}$ & $a_{32}$ & $p_{32}$ \\
\hline- & - & - & - & - \\
\hline $\mathrm{n}$ & $a_{n 1}$ & $p_{n 1}$ & $a_{n 2}$ & $p_{n 2}$ \\
\hline
\end{tabular}

Table 1

Mathematically, the problem is stated as: Minimize $R\left(S_{k}\right)=\sum A_{i 1} \times C_{1}+$ $U_{2}\left(S_{k}\right) \times C_{2}$.

Subject to constraint: Rental Policy $(\mathrm{P})$. Our objective is to minimize rental cost of machines while minimizing the utilization time.

\section{Assumptions}

1. Two jobs cannot be processed on a single machine at a time.

2. Jobs are independent to each other.

3. Per-emption is not allowed i.e. once a job started on a machine, the process on that machine cannot be stopped unless the job is completed.

4. Either the processing time of the $i^{\text {th }}$ job of machine $M_{1}$ is longer than the expected processing time of $j^{\text {th }}$ job on machine $M_{2}$ or the processing time $i^{\text {th }}$ job on machine $M_{1}$ is shorter than the expected processing time of $j^{\text {th }}$ job on machine $M_{2}$ for all i,j. either $A_{i 1} \geq A_{i 2}$ or $A_{i 1} \leq A_{i 2}$

5. $\sum p_{i 1}=1, \sum p_{i 2}=1$ for all i.

6. Let $\mathrm{n}$ jobs be processed through two machines $M_{1}$ and $M_{2}$ in order $M_{1} M_{2}$ and in order $M_{2} M_{1}$.

7. Machine break down is not considered.

\section{Theorem}

If for all $A_{i 1} \leq A_{j 2}$ for all $i, j, i \neq j$, then $k_{1}, k_{2} \ldots k_{n}$ is a monotonically decreasing sequence, where $K_{n}=\sum A_{i 1}-\sum A_{i 2}$.

Proof. Let $A_{i 1} \leq A_{j 2}$ for all i, j, i $\neq$ j i.e., $\max A_{i 1} \leq \min A_{j 2}$ for all i, $\mathrm{j}, \mathrm{i} \neq$ j Let $K_{n}=\sum A_{i 1^{-}} \sum A_{i 2}$.

Therefore, we have $k_{1}=A_{11}$. 
Also

$$
K_{2}=A_{11}+A_{21}-A_{12}=A_{11}+\left(A_{21}-A_{12}\right) \leq A_{11}
$$

(as $A_{21} \leq A_{12}$ ), so $K_{1} \leq K_{2}$.

Now,

$$
\begin{aligned}
K_{3}=A_{11}+A_{21}+A_{31}-A_{12}-A_{22}=A_{11}+A_{21} & -A_{12}+\left(A_{31}-A_{22}\right) \\
& =K_{2}+\left(A_{31}-A_{22}\right) \leq K_{2}
\end{aligned}
$$

(as $A_{31} \leq A_{22}$ ).

Therefore, $K_{3} \leq K_{2} \leq K_{1}$ or $K_{1} \geq K_{2} \geq K_{3}$.

Continuing in this way, we can have $K_{1} \geq K_{2} \geq K_{3} \ldots \ldots . K_{n}$, a monotonically decreasing sequence.

Corollary 1. The total rental cost of machines is same for all the sequences if $A_{i 1} \leq A_{j 2}$ for all $i, j, i \neq j$.

Proof. The total elapsed time $T(S)=\sum A_{i 2}+K_{1}=\sum A_{i 2}+A_{11}$.

It implies that under rental policy $\mathrm{P}$ the total elapsed time on machine $M_{2}$ is same for all the sequences thereby the rental cost of machines is same for all the sequences.

\section{Theorem}

If $A_{i 1} \geq A_{j 2}$ for all $i, j, i \neq j$, then $k_{1}, k_{2} \ldots k_{n}$ is a monotonically increasing sequence, where $K_{n}=\sum A_{i 1}-\sum A_{i 2}$

Proof. Let $K_{n}=\sum A_{i 1}-\sum A_{i 2}$.

Let $A_{i 1} \geq A_{j 2}$ for all $i, j, i \neq j$, i.e. $\min A_{i 1} \geq \max A_{j 2}$ for all $i, j, i \neq j$.

Here $k_{1}=A_{11}, K_{2}=A_{11}+A_{21}-A_{12}=A_{11}+\left(A_{21}-A_{12}\right) \geq k_{1}\left(\right.$ as $A_{21} \geq$ $A_{12}$ ) so $K_{2} \geq K_{1}$

Now, $K_{3}=A_{11}+A_{21}+A_{31}-A_{12}-A_{22}=A_{11}+A_{21}-A_{12}+\left(A_{31}-A_{22}\right)$

$=K_{2}+\left(A_{31}-A_{22}\right) \geq K_{2}\left(\right.$ as $\left.A_{31} \geq A_{22}\right)$

Hence, $K_{3} \geq K_{2} \geq K_{1}$.

Continuing in this way, we can have $K_{1} \leq K_{2} \leq K_{3} \ldots \ldots \leq K_{n}$, a monotonically increasing sequence.

Corollary 2. The total elapsed time of machines is same for all the possible sequences.

Proof. The total elapsed time 


$$
\begin{aligned}
& T(S)=\sum A_{i 2}+K_{n}=\sum A_{i 2}+\left(\sum A_{i 1}-\sum A_{i 2}\right) \\
&=\sum A_{i 1}+\left(\sum A_{i 2}-\sum A_{i 2}\right)=\sum A_{i 1}+A_{n 2}
\end{aligned}
$$

Therefore total elapsed time of machines is same for all the sequences if $A_{i 1} \geq$ $A_{j 2}$ for all $i, j, i \neq j$.

\section{Algorithm}

Step 1: Calculate the expected processing time $A_{i j}=a_{i j} \times p_{i j} \forall i, j$.

Step 2: Take the equivalent job $(k, m)$ and calculate the processing time $G_{\beta}$ and $H_{\beta}$ on guide lines of Maggu and Dass (1982) as follows:

$G_{\beta}=G_{k}+G_{m}-\min \left(G_{m}, H_{k}\right)$ and $H_{\beta}=H_{k}+H_{m}-\min \left(G_{m}, H_{k}\right)$

Step 3: Define a new reduced problem with processing times $G_{i}$ and $H_{i}$ as defined in step 1 and jobs $(k, m)$ are replaced by single equivalent job $\beta$ with processing time $G_{\beta}$ and $H_{\beta}$ as defined in step 2.

Step 4:Check the condition either $G_{i} \geq H_{j}$ or $G_{i} \leq H_{j}$, for all $i$ and $j, i \neq$ $j$ if conditions hold good, go to step5, else the results will coincides with [5].

Step 5: Obtain the job $J_{1}$ (say) having maximum processing time an first machine and job $J_{n}$ (say) having minimum processing time an second machine.

Step 6: If $J_{1} \neq J_{n}$ then put $J_{1}$ on the first position and $J_{n}$ as the last position and go to step 9, Otherwise go to step 7.

Step 7: Take the difference of processing time of job $J_{1}$ on first machine from job $J_{2}$ (say) having next maximum processing time on first machine. Call this difference as $G_{1}$. Also, take the difference of processing time of job $J_{n}$ on second machine from job $J_{n-1}$ (say) having next minimum processing time on second machine. Call the difference as $G_{2}$.

Step 8: If $G_{1} \neq G_{2}$ put $J_{n}$ on the last position and $J_{2}$ on the first position otherwise put $J_{1}$ on first position and $J_{n-1}$ on the last position.

Step 9: Arrange the remaining (n-2) jobs between first job and last job in any order, thereby we get the sequences $S_{1}, S_{2}, S_{3}, \ldots, S_{r}$ (when the order of machines is $M_{1} \rightarrow M_{2}$ and the sequences $S_{1}^{\prime}, S_{2}^{\prime}, S_{3}^{\prime}, \ldots, S_{r}^{\prime}$ (when the order of machines is $M_{2} \rightarrow M_{1}$ ).

Step 10: Compute the total completion time $C T\left(S_{k}\right)$ and $C T\left(S_{k}^{\prime}\right)$ by computing in out table for sequences $S_{k}$ and $S_{k}^{\prime}(k=1,2, \ldots, r$.$) .$ 
Step 11: Calculate utilization time $C T\left(S_{k}\right), U_{2}\left(S_{k}\right)$ and $U_{1}\left(S_{k}^{\prime}\right)$ of second machine as follows:

$U_{2}\left(S_{k}\right)=C T\left(S_{k}\right)-A_{11}\left(S_{k}\right) ; k=1,2, . \quad r . \quad$ and $U_{1}\left(S_{k}^{\prime}\right)=C T\left(S_{k}^{\prime}\right)-A_{12}\left(S_{k}^{\prime}\right)$; $k=1,2, . r$.

Step 12: Find rental cost $R\left(S_{1}\right)=\sum A_{i 1}\left(S_{1}\right) \times C_{1}+U_{2}\left(S_{1}\right) \times C_{2}$, where $C_{1}$ and $C_{2}$ are the rental cost per unit time of machine $M_{1}$ and $M_{2}$ respectively.

Step 13: Find rental cost $R\left(S_{1}^{\prime}\right)=\sum A_{i 2}\left(S_{1}^{\prime}\right) \times C_{2}+U_{2}^{\prime}\left(S_{1}^{\prime}\right) \times C_{1}$ where $C_{1}$ and $C_{2}$ are the rental cost per unit time of machine $M_{1}$ and $M_{2}$ respectively.

Step 14: Find $R(S)=\min \left(R\left(S_{1}\right), R\left(S_{1}^{\prime}\right)\right)$.

\section{Numerical Illustration}

Consider 6 jobs, 2 machines problem to minimize the rental cost, processing time associated with their respectively probabilities as given in following table and job 2,5 are to be processed as a group job $(2,5)$. The rental cost per unit time for machines $M_{1}$ and $M_{2}$ are 10 and 5 units respectively. Our objective is to obtain optimal schedule and order of machines to minimize the total production time subject to minimization of the rental cost of the machines, under the rental policy p.

\begin{tabular}{|c|c|c|c|c|}
\hline jobs & \multicolumn{2}{|c|}{ Machine } & $M_{1}$ & \multicolumn{2}{l|}{ Machnie } & $M_{2}$ \\
\hline $\mathrm{i}$ & $a_{i 1}$ & $p_{i 1}$ & $a_{i 2}$ & $p_{i 2}$ \\
\hline 1 & 40 & 0.2 & 75 & 0.2 \\
\hline 2 & 30 & 0.3 & 80 & 0.2 \\
\hline 3 & 45 & 0.1 & 85 & 0.2 \\
\hline 4 & 35 & 0.2 & 90 & 0.2 \\
\hline 5 & 25 & 0.1 & 95 & 0.1 \\
\hline 6 & 50 & 0.1 & 100 & 0.1 \\
\hline
\end{tabular}

Table 2

Solution. As per Step 1: The expected processing time for machines $M_{1}$ and $M_{2}$ is as follow 


\begin{tabular}{|c|c|c|}
\hline job & $A_{i 1}$ & $A_{i 2}$ \\
\hline 1 & 8.0 & 15.0 \\
\hline 2 & 9.0 & 16.0 \\
\hline 3 & 4.5 & 17.0 \\
\hline 4 & 7.0 & 18.0 \\
\hline 5 & 2.5 & 9.5 \\
\hline 6 & 5.0 & 10.0 \\
\hline
\end{tabular}

Table 3

when the order of machines is $M_{1} \rightarrow M_{2}$.

As per Step 2: Here $\beta=(2,5), G_{\beta}=G_{2}+G_{5}-\min \left(G_{5}, H_{2}\right)=9.0$ and $H_{\beta}=H_{2}+H_{5}-\min \left(G_{5}, H_{2}\right)=23.0$

\begin{tabular}{|c|c|c|}
\hline job & $G_{i}$ & $H_{i}$ \\
\hline 1 & 8.0 & 15.0 \\
\hline$\beta$ & 9.0 & 23.0 \\
\hline 3 & 4.5 & 17.0 \\
\hline 4 & 7.0 & 18.0 \\
\hline 6 & 5.0 & 10.0 \\
\hline
\end{tabular}

Table 4

As per step $4 G_{i} \leq H_{i}$ for all $\mathrm{i}, \mathrm{j}, \mathrm{i} \neq \mathrm{j}$. Also $\max G_{i}=9.0$ which is for job $\beta$ i.e. $J_{1}=\beta$ And $\min H_{i}=10.0$ which is for job 3 i.e. $J_{n}=6$ Since $J_{1} \neq J_{n}$.

As per Step 6 , job $\beta$ will be on first position and job 6 will be on the last position. Therefore as per step 9 the possible optimal sequences are $S_{1}: \beta-1$ - 3 - 4 - $6, S_{2}: \beta-3-1-4-6, S_{3}: \beta-1-4-3-6, S_{4}: \beta-3-4-1-6, S_{5}: \beta$ $-4-1-3-6, S_{6}: \beta-4-3-1-6$

\begin{tabular}{|c|c|c|}
\hline $\begin{array}{c}\text { job } \\
\mathrm{i}\end{array}$ & $\begin{array}{c}\text { Machine } M_{1} \\
\text { In-Out }\end{array}$ & $\begin{array}{c}\text { Machine } M_{2} \\
\text { In-Out }\end{array}$ \\
\hline 2 & $0-9.0$ & $9.0-25.0$ \\
\hline 5 & $9.0-11.5$ & $25.0-34.5$ \\
\hline 1 & $11.5-19.5$ & $34.5-49.5$ \\
\hline 3 & $19.5-24.0$ & $49.5-66.5$ \\
\hline 4 & $24.0-31.0$ & $66.5-84.5$ \\
\hline 6 & $31.0-36.0$ & $84.5-94.5$ \\
\hline
\end{tabular}

Table 5

The total elapsed time is same for all these 6 possible sequence $S_{1}, S_{2}$, 
$S_{3}, S_{4}, S_{5}, S_{6}$ The in- -out table for any of these sequences $S_{1}, S_{2}, S_{3}, S_{4}, S_{5}$, $S_{6}$ Say for $S_{1}: 2 \quad 5 \quad 1 \quad 3 \quad 4 \quad 6$

Therefore the total elapsed time $=C T\left(S_{1}\right)=94.5$ units and utilization time for $M_{2}=U_{2}\left(S_{1}\right)=85.5$ units Also $\sum A_{i 1}=36.0$ and rental cost $R\left(S_{1}\right)=36.0$ $10+85.55=787.5$ units, when the order of machines is $M_{2} \rightarrow M_{1}$.

As per Step 2: Here $\beta=(2,5) G_{\beta}=G_{2}+G_{5}-\min \left(G_{5}, H_{2}\right)=16.5$ and $H_{\beta}$ $=H_{2}+H_{5}-\min \left(G_{5}, H_{2}\right)=2.5$.

As per Step 3: New reduced problem is as follows

\begin{tabular}{|c|c|c|}
\hline job & $G_{i}$ & $H_{i}$ \\
\hline 1 & 15.0 & 8.0 \\
\hline$\beta$ & 16.5 & 2.5 \\
\hline 3 & 17.0 & 4.0 \\
\hline 4 & 18.0 & 7.0 \\
\hline 6 & 10.0 & 5.0 \\
\hline
\end{tabular}

Table 6

As per step $4 G_{i}$ and $H_{j}$ for all i, j. Also $\max G_{i}=18.0$ which is for job 4 and $\min H_{i}=2.5$ which is for job $\beta$ i.e. $J_{n}=\beta$ Since $J_{1} \neq J_{n}$.

As per step 6 , job 4 will be on first position and job $\beta$ will be on the last position.

As per step 9 the possible optimal sequences are $S_{1}^{\prime}: 4-1-5-3-\beta, S_{2}^{\prime}: 4-1-3-$ $5-\beta, \ldots, S_{6}^{\prime}: 4-3-6-1-\beta$

The total elapsed time is same for all these 6 possible sequence $S_{1}^{\prime}, S_{2}^{\prime}, \ldots, S_{6}^{\prime}$ The in- -out table for any of these sequences $S_{1}^{\prime}, S_{2}^{\prime}, \ldots, S_{6}^{\prime}$ Say for $S_{1}^{\prime}: 4-3-1-6-2-5$.

\begin{tabular}{|c|c|c|}
\hline $\begin{array}{c}\text { job } \\
\mathrm{i}\end{array}$ & $\begin{array}{c}\text { Machine } M_{2} \\
\text { In-Out }\end{array}$ & $\begin{array}{c}\text { Machine } M_{1} \\
\text { In-Out }\end{array}$ \\
\hline 4 & $0-18$ & $18-25.0$ \\
\hline 3 & $18-35$ & $35-39.5$ \\
\hline 1 & $35-50$ & $50-58$ \\
\hline 6 & $50-60$ & $60-65$ \\
\hline 2 & $60-76$ & $76-85$ \\
\hline 5 & $76-85.5$ & $85.5-88.0$ \\
\hline
\end{tabular}

Table 7

Therefore the total elapsed time $=C T\left(S_{1}^{\prime}\right)=88.0$ units and utilization time for $M_{1}=U_{1}\left(S_{1}^{\prime}\right)=70.0$ units Also $\sum A_{i 2}=85.5$ and rental cost $R\left(S_{1}^{\prime}\right)=1127.5$ units $R(S)=\min \left(R\left(S_{1}\right), R\left(S_{1}^{\prime}\right)\right)=787.5$ units $=R\left(S_{1}\right)$. 
We conclude that optimal schedule $S_{1}^{\prime}: 4-3-1-6-2-5$, which is for order Machine $M_{1}$ to Machine $M_{2}$ gives minimum rental cost.

\section{Remarks}

1. If the assumption 4 is not considered the result tally with [5].

2. Equivalent job formulation is associative and non commutative in nature.

3 . The study on $n^{*} 2$ open shop scheduling may be further extended by including various parameters such as weightage of jobs, transportation etc.

4. The study may further be extended for $n$ job 3 machine open shop problem.

5. If we solve the same problem by Johnson[1] method we get optimal sequence as $\mathrm{S}=3-6-4-1-\beta=3-6-4-1-2-5$ for $M_{1}$ to Machine $M_{2}$

This gives rental cost $\mathrm{R}(\mathrm{S})=837.5$ Units And for order $M_{2}$ to Machine $M_{1}$ we get optimal sequence as $\mathrm{S}=1-4-6-3-\beta=1-4-6-3-2-5$. which gives $\mathrm{R}(\mathrm{S})=1157.5$. Thus rental cost is minimum for $M_{1}$ to Machine $M_{2}$.

\section{Conclusion}

The algorithm proposed in this paper to minimize the rental cost of machines gives an optimal sequence having minimum rental cost of machines. The algorithm proposed by Johnson [1] to find an optimal sequence to minimize the makespan / total elapsed time is not always corresponds to minimum rental cost the machines. Hence proposed algorithm is more efficient to minimize the rental cost of machines under a specified rental policy. We conclude that ours algorithm gives better result.

\section{References}

[1] Johnson, S.M. , Optimal two and three stage Production Schedule with set-up times included. Nav Res Log.Quart., Vol. 1, 1954, pp.61-64.

[2] Ignall, E., and Schrage, L. , Application of the branch and bound technique to some flow shop scheduling problems . Operation Research, Vol. 13, 1965, pp.400-412.

[3] Bagga, P.C., Sequencing in a rental situation, Journal of Canadian Operation Research Society, Vol.7, 1969, pp.152-153. 
[4] Gupta, J.N.D, Optimal Schedule for specially structured flow shop, Naval Res. Logist. Quart., Vol.22,No.2, 1975, pp.255-269.

[5] Gupta D.and Singh, T.P., On job block open shop scheduling, the processing time associated with probability.,J. Indian Soc. Stat. Opers. Res., Vol.XXVI No.1-4,2005,pp.91-96.

[6] Harbans, Lal and Maggu, P.L., On job block open shop scheduling problem, PAMS, Vol.XXIX, 1989, pp.45-51.

[7] Gonzalez, T. and Sahni, S. , Open shop scheduling to minimize nish time, J. Assoc. Comput., March. 23, 1976, pp.665-679.

[8] Singh, T. P. and Gupta D. , Minimizing rental cost in two stage flow shop, the processing time associated with probabilities including job block, Reflections de ERA, Vol. 1, No.2, 2005, pp.107-120.

[9] Maggu, P.L.and Das G. , Equivalent jobs for job block in job scheduling, Opsearch, Vol.14, No.4,1977, pp.277-281.

[10] Yoshida and Hitomi ,Optimal two stage production scheduling with set up times separated, AIIE Transactions,Vol. 11,No.3, 1979, pp.261-269.

[11] Gupta, J.N.D, Two stage hybrid flow shop scheduling problem, J. Ope. Res. Soc., Vol.39,No.4, 1988, pp.359-364.

[12] Singh, T. P. , On n2 shop problem involving job block. Transportation times and Break-down Machine times, PAMS, Vol. XXI, No.2, 2005, pp.1.

[13] V.J. Rayward-Smith,and D. Rebaine, Open-shop scheduling with delays, Theoret. Inform. Appl.,1992, 439-448.

[14] V.A. Strusevich, Two-machine open-shop scheduling problem with setup, processing and removal times separated, Comput. Oper. Res.,1993, 597611.

[15] Amico, M.,Dell, Shop problems with two machines and time lags, Oper. Res.,1996,Vol.44, 777-787..

[16] V.A. Strusevich, An open-shop scheduling problem with a non-bottleneck machine, Oper. Res. Lett.,1997,Vol.21, 11-18.

[17] Rebaine,D.,and Strusevich,V.A., Two-machine open shop scheduling with special transportation times, CASSM Rand D,1998,Vol.15, University of Greenwich, London, UK. 
[18] Anup, On two machine flow shop problem in which processing time assumes probabilities and there exists equivalent for an ordered job block, JISSOR,2005, Vol. XXIII,No.1-4.

[19] T.P.Singh, Rajender kumar and Gupta Deepak, Optimal three stage production schedule the processing time and set times associated with probabilities including job block criteria, Acta Ciencia Indica, Proceedings of National Conference FACM, 2005.

[20] Gupta D.,Sharma,S., and Shashi B., Optimal three stage production schedule the processing time and set times associated with probabilities including job block criteria, Specially Structured Two Stage Flow Shop Scheduling To Minimize the Rental Cost, International Journal of Emerging trends in Engineering and Development, Vol. 1, No. 2, 2005, pp.206-215. 